\title{
Unilateral Male Breast Lymphangioma: A Case Report with Concern for Reconstruction
}

\section{Brownstone $\mathrm{N}^{*}$ and Otterburn D}

Department of General Surgery, New York Presbyterian-Weill Cornell Medical Center, USA

*Corresponding author: Nicholas Brownstone, NewYork-Presbyterian Hospital, 515 Spruce Street San Francisco, CA 94118, United States, Tel: 9089171849; Email: nickbrownstone34@ gmail.com

\section{Case Report \\ Volume 4 Issue 1}

Received Date: June 10, 2020

Published Date: June 26, 2020

DOI: $10.23880 /$ ijtps- 16000147

\section{Abstract}

Lymphatic malformations are congenital malformations that are usually evident at birth and rarely manifest in adolescence. The breast is an extremely rare site for these masses and only a handful of them have been described in the literature. Surgical resection is the only way to potentially cure this disorder. Here we describe a case of a 15-year-old male presenting with a large lymphangioma with a discussion on reconstruction to minimize recurrence and maximize an aesthetic result.

Keywords: Lymphangioma; Reconstruction; Pediatric breast masses

\section{Introduction}

Lymphatic malformations comprise anomalous channels, vesicles or pouches filled with lymphatic fluid and can be described as microcystic, macrocystic or combined (micromacrocystic). Most are evident at birth or are found before 2 years of age. They rarely manifest in adolescence. These malformations are usually described in the cervicofacial region, extremities, visceral organs or skeletal structure. The breast is an extremely rare site for these masses and only a handful of them have been described in the literature. An even smaller subset of these has been described in children. One review of the literature only found 17 cases of breast lymphangiomas and of these only 4 were in children [1]. Surgical resection is the only way to potentially cure this disorder. Here we describe a case of a 15-year-old male presenting with a large lymphangioma with a discussion on reconstruction to minimize recurrence and maximize an aesthetic result.

\section{Case}

A 15-year-old male with no significant past medical history or medication use was referred to pediatric surgery by his pediatrician after an aesthetic concern over a left breast mass. The mass has been steadily growing for past 5 years without evidence of induration, erythema or local lymph node involvement. He denied any pain, drainage or variations in size but reported that the mass turned blue at times. Upon physical exam, a large and fluctuant mass was found in the lateral left breast without evidence of skin changes. A breast ultrasound revealed a retroareolar cystic structure with minimal debris and anterior nodularity suggesting a complex cyst. An MRI, with and without contrast, demonstrated a predominantly $\mathrm{T} 2$-hyperintense lesion centered entirely in the subcutaneous fat of the left anterior chest wall. This read was most compatible with a lymphangioma. Cytology obtained from fine needle aspiration was consistent with benign contents. Non-invasive treatment by aspiration was attempted but the mass recurred.

As a result, pediatric surgery planned to excise the mass with plastic surgery consultation for approach and closure. An inframammary incision was planned, as the mass was considered too big for a periareolar approach. In the operating room, a $6 \mathrm{~cm}$ long IMF incision was made in the left breast, dissecting down through the skin and subcutaneous tissues, identifying the pectoralis major muscle and then developing a retro-mass plane which involved the pectoralis major muscle, fascia and also the subcutaneous plane. Pediatric 


\section{International Journal of Transplantation \& Plastic Surgery}

surgery circumscribed and removed an approximately $10 \mathrm{~cm}$ lymphangioma that was densely adherent to the nipple and which seemed to take the place of the entire breast area. The rest of the mass was then excised off the nipple by inverting it, sharply excising it to prevent any nipple necrosis. The pocket was then irrigated and the $6 \mathrm{~cm}$ long incision was then closed in layers after widely undermined to allow for a tension-free repair. At six months post-op, the scar healed well without evidence of recurrence on ultrasound.

\section{Discussion}

The differential diagnosis of pediatric breasts masses includes many types of pathologies. While cancer is a concern in the adult population, parents can be reassured that breast cancer in the pediatric population is almost never seen with an incidence below one in a million patients. As a result, almost all pediatric breast masses are benign masses such as cyst, hematoma, mastitis/abscess and galactocele. The most common benign solid mass is a fibroadenoma. Clinical history and physical exam is an important diagnostic modality and it is important to remember that normal anatomic structures can sometimes mimic breast masses [2].

Macrocystic lymphangiomas, formerly known as cystic hygroma, are congenital hamartomatous malformations of the lymphatic system that are thought to form due to a misconnection between lymphatic and venous systems or sequestration of small lymph channels from the main network [3]. They are not true neoplasms and have a predilection for the head and neck region. More than $90 \%$ of cystic hygroma cases are diagnosed by the age of 2 ; in almost all patients (>90\%) the lesion is located in the neck or axilla specifically [4]. The breast is a rare place for these tumors to be found. In one study of 22 children treated for lymphangioma over a ten-year period, only one was found in the breast [5]. The average age of diagnosis is 3.3 years [6]. In another study, one study of 74 pediatric patients who presented with various types of breast masses over a 13-year period found no diagnoses of lymphangioma [7].

Mammography, ultrasonography and magnetic resonance imaging (MRI) can be used to help diagnose lymphangiomas. On mammography, they appear as diffuse or localized dense masses with lobulated margins and no macro- or microcalcifications are present [8]. Ultrasonography usually shows a multi-loculated, hypoechoic, cystic mass with linear septa that contain solid elements originating from the cyst walls or septa [9]. The benefit of ultrasound is that it is cheap and readily available without risk of radiation exposure. The disadvantage is that it is poor at defining the precise margins of the mass. MRI is the modality of choice for diagnosis and evaluation of lymphangiomas in the breast and it is usually seen as a septated mass of high T2 weighted signal intensity with variable enhancement from septae only [10].

Sclerotherapy has been used as a major treatment modality and large cysts can be treated with aspiration of lymph and instillation of scerlosing agents such as doxycycline, sodium tetradecyl sulfate or OK-432 (lyophilized incubation mixture of group A Streptococcus pyogenes of human origin). While primary surgical excision, when compared to sclerotherapy, is the most successful technique, complications after its use are more serious such as damage to surrounding nerves and vessels. However, sclerotherapy results in inflammation and fibrosis and makes any subsequent excision much more difficult. One theoretical benefit of sclerotherpy versus surgical excision is that surgery can result in a poorer cosmetic result due to scarring [11]. Sometimes, complete excision may not be possible because of close proximity to vital structures and disfigurement caused by extensive dissection of a very largesized tumor or when saving the breast tissue is of concern as in girls and young females [12]. Despite this, it is imperative to perform as thorough of a resection as possible given these restrictions due risk of recurrence. When considering points above, we advocate for primary excision and reserve sclerotherapy for recurrent cases or poor surgical candidates.

Reconstructive techniques are always important to consider but they especially relevant in the setting of multiple areas of disease or where extirpation is around an area of aesthetic concern. Reconstructive and aesthetic planning begins with the proper incisional and excisional approach. Mastectomy should be avoided due to the concern for developing breast tissue but may be considered for advanced disease. Mukhopadhyay, et al. successfully described using mastectomy in treating bi-lateral pediatric breast tumors measuring $22 \mathrm{~cm}$ by $18 \mathrm{~cm}$ [13]. If mastectomy is to be attempted, nipple-sparing mastectomy is the procedure of choice in the pediatric population. Sosin, et al. describes removing a large $12 \mathrm{~cm}$ lateral fibroadenoma of the breast with a circumareolar incision with short lateral extension in a 13-year-old female [14]. While this has poor visualization and leaves a short scar on the lateral breast tissue, the author reports that this technique allows in situ breast tissue to fill the void and re-create the normal breast contour, which maximizes the aesthetic result. We chose an inframammary fold approach due to the size of the tumor and need for complete visualization as any residual lymphagiomatous mass is at high risk for recurrence. Although a circumareolar incision may give an acceptable result, avoiding incisions on the male breast is optimal. The IMF incision avoids possible painful incisions near the nipple, avoids tethering and may reduce nipple anesthesia, necrosis and pain. 


\section{International Journal of Transplantation \& Plastic Surgery}

\section{Conclusion}

Although multiple treatments modalities and excisional methods exist, there is a paucity of primary source literature comparing and contrasting their effectiveness and aesthetic outcomes. When dealing with lymphangiomas, it is paramount to obtain good visualization and take care as to not leave any residual tissue. While this presentation was extremely rare, these techniques are applicable to many other kinds of pediatric breast masses. It is integral for primary care physicians, breast surgeons, general surgeons, pediatric surgeons and plastic surgeons to be aware of pediatric breast tumors and options for treatment to achieve the best reconstructive and aesthetic outcome.

\section{References}

1. Gupta SS, Singh O (2011) Cystic lymphangioma of the breast in an 8-year-old boy: Report of a case with a review of the literature. Surg Today 41: 1314.

2. Yiming G, Mansi A Saksena, Elena F Brachtel, Deborah C terMeulen, Elizabeth A (2015) How to approach breast lesions in children and adolescents. Eur J Radiol 84(7): 1350-1364.

3. Singh O, Singh Gupta S, Upadhyaya VD, Sharma SS, Lahoti BK, et al. (2009) Cystic lymphangioma of the breast in a 6-year-old boy. J Pediatr Surg 44(10): 2015-2018.

4. Chung SY, Oh KK, Kim DJ (2003) Mammographic and sonographic findings of a breast cystic lymphangioma. J Ultrasound Med 22(3): 307-309.

5. Al-Salem AH (2004) Lymphangiomas in infancy and childhood. Saudi Med J 25(4): 466-469.
6. Alqahtani A, Nguyen LT, Flageole H, Shaw K, Laberge JM (1999) 25 years' experience with lymphangiomas in children. J Pediatr Surg 34(7): 1164-1168.

7. West KW, Rescorla FJ, Scherer LR, Grosfeld JL (1995) Diagnosis and treatment of symptomatic breast masses in the pediatric population. J Pediatr Surg 30(2): 182187.

8. Balaji R, Ramachandran K (2010) Cystic Lymphangioma of the Breast: Magnetic Resonance Imaging Features. Breast Care 5(4): 250-252.

9. Chung EM, Cube R, Hall GJ, González G, Thomas Stocker J, et al. (2009) Breast Masses in Children and Adolescents: Radiologic-Pathologic Correlation. Radio Graphics 29(3): 907-931.

10. Ko KH, Kim EK, Kang HY, Youk JH (2009) Cavernous lymphangiomas of the breast mimicking breast cancer. J Ultrasound Med 28(7): 973-976.

11. Okazaki T, Iwatani S, Yanai T, Kobayashi H, Kato Y, et. al. (2007) Treatment of lymphangioma in children: our experience of 128 cases. J Pediatr Surg 42(2): 386-389.

12. Okoro PE, Anyaeze CM, Ngaikedi C (2009) Recurrent lymphangioma: what are the treatment options. Afr J Paediatr Surg 6(1): 44-46.

13. Mukhopadhyay M, Patra R, Mondal S, Ghosh A, Ray AK (2009) Bilateral giant juvenile fibroadenoma of breasts. J Indian Assoc Pediatr Surg 14(2): 68-69.

14. Sosin M, Feldman E (2012) Giant juvenile fibroadenoma: A case and review of novel modalities in treatment. Breast Disease 34(1): 35-38. 